Invisible Conflicts:

\title{
The Challenges of Design on a Planetary Scale
}

BANI BRUSADIN

Elisava, Barcelona School of Design and Engineering (UVic-UCC)

UB - University of Barcelona

LAURA BENÍTEZ

Elisava, Barcelona School of Design and Engineering (UVic-UCC)

UAB - Autonomous University of Barcelona

Escola Massana, Art and Design Centre

TEMES DE DISSENY \#37
KEYWORDS

Infrastructures, Regimes of Visibility, Design on a Planetary Scale, Agency of Non-Human Actors Technologically Mediated Power Structures, Dissent and Resistance.

LICENSE

\section{BY-NC-ND}

HOW TO CITE

Brusadin, Bani, and Laura Benítez Valero. 2021. "Invisible Conflicts: The Challenges of Design on a Planetary Scale". Temes de Disseny 37: 8-17.

EDITORIAL
What interests us is to glimpse

For example, to what For example, the proliferation The experience of the interconnected planet is the experi- of online ence of an artificial and often sinister totality. It is the result distributiolf-employed or self-employed in name only - roam with its topat artisected in invisible substem and the city in patern that cannot be predicted by urban layers, which in turn compete with one another according to ners? How is urban life impacted by the fact that a growing the irreoular logics of rival interests, incompatible designs, number of actors make very concrete decisions motivated by human habits and rhythms, forms of resistance, errors, their interactions with and on private platforms (designed to collateral effects and accidents. For example, modern na- allow no role other than user)? The very notions of labour, tional borders and jurisdictions are being crossed by data the means of production, value and capital accumulation packets and their processing provoking theoretical and are being subjected to a profound tectonic movement at the practical conflicts due to the redefinition of the very idea of moment, when an activity such as driving a vehicle which sovereignty, its subject and its object (Bratton 2016). The is generally considered unskilled labour without added valpersonal sphere is diluted into platforms designed for bil- ue, has now become a fundamental process for producing lions of users to express themselves with different degrees information, extracting intelligence and training automated of freedom, but their autonomy collapses and vanishes in agents on a global scale (Pasquinelli and Joler 2020). How do infrastructures on a literally unimaginable scale, rigidly data - not so much at the individual, subjective or particular proprietary but ungovernable, automated and entropic, scale, but in terms of involuntary, mechanically generated with volatile masses of users yet simultaneously sustained and aggregated data - question social processes developed by global oligopolies, strategic investments and geopoliti- over decades or centuries such as urban planning, trade been penetrated by processes of quantification, modelling, tlefields between the extraction of resources for human

It is evident that this is fully part of the realm of There, products are not organised according to a commercial users' consumer behaviour on the platform, recorded and analysed practically in real time; the operating patterns of all the actors in the distribution and logistics chains; and even indirectly, a flexible business model in which Amazon, as a producer, is able to instantly access profitable niche markets instant tracking of independent sellers' successes or failures ments, but there is an important slippage: workers follow the 2020) built by and for real-time data processing. They use to the evolution of different factors - is to control productivity
by drastically minimising workers' need for intelligence, deciby drastically minimising workers' need for intelligence, decision-making power and intuition. Amazon fulfilment centres are prime examples about which rather little is known as
they are indeed impenetrable spaces in which guided tours they are indeed impenetrable spaces in which guided tours
serve promotional purposes at the very best. These spaces serve promotional purposes at the very best. These spaces
become understandable thanks to indirect operations such themselves or the reverse engineering carried out through cal tensions. ${ }^{1}$ Even the realms of biology and geology have exploitation and reformation that transform them into batactivities and the production of expanter-power (Gabrys 2016). design if we look, for example, at the operation of such a quotidian object today as an Amazon distribution centre. or functional taxonomy, nor even by physical characteristics that are visible at a quick glance. On the contrary, in these warehouses, other places and other bodies come into play: as soon as revenue opportunities are detected (thanks to on the platform). Of course, the organisation of a designed space takes into account the efficiency of workers' moveinstructions of the management system, which guides them through an "operational landscape" (Brenner and Katsiki. terminals and are terminals themselves. The goal of this architecture - designed, at least in part, to dynamically adap as, for example, oral accounts from self-organised worker B. BRUSADIN / L. BENÍTEZ

nionism or the very notion of political jurisdiction? In a world where cognitive stimuli overload actuly pushes people's attention toward a plethora of details and short-term reactions, the colossal proportions of quantication and prediction procedures are actually leading us the opposite side of abstraction. The responsible players production and storage on a massive scale, but also dynamic processing that produces interpretive paterns that are invisible to "users" (ormer citizens), not more incompre( enchen systems (n) Tadically new manners: How do you make a decision? In 作 ten subjective worlds and he material planet? What does twe a . 作

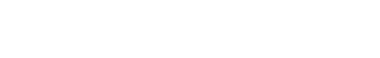
the Dnly addressed invisible conflicts but have also used the pers nd non-human otherness. Some of the leading figures in the eld in this case within speculative design, are Dunne and projects like Foragers ${ }^{2}$ and apply it, ironically, to the agent responsible for the ecological debacle: the human agent. A this drift towards abstraction are the processes of data Raby, who subvert solutionist technological mediation with 
crete production and extraction system, that is to say, cap- "The greatest danger for me is the danger of the autonomous
italist. In this issue of Temes de Disseny, the operational and and monolithic subject that seeks to establish limits and italist. In this issue of Temes de Disseny, the operational and and monolithic subject that seeks to establish limits and 2021) 2021) goes precisely in this direction by deconstructing the refuses to recognise its fundamentally social character and extractive processes implicit in the creation of the datasets its interdependence. And it seems to me that no solid ethics to train facial recognition systems.

ing other ways of doing and making with other non-human agents as well as to rethinking issues such as agency through materiality. This issue addresses the practice of design as a and becoming. Practices and projects are also unfolded to help us understand relatively invisible conflicts that are present, for example, in contemporary theory. Continuing to support dualistic structures such as the human and the non-human, the artificial and the natural, or the organic and the inorganic is becoming increasingly complex. Undoubtedly, thinkers like Bruno Latour (2005) have long argued that what we call the world is so fabricated and that tools and technologies are so ubiquitous that we cannot meaningfully separate the human from the non-human. However, it should be noted that this impossibility of significant separation it is precisely this equality in differences that challenges human agents to commit themselves to other co-beings,
other relationships where the scales, affects and affectations re diverse. This question was already present in moder philosophy. Baruch Spinoza (re)defined human agency, relocated it and repositioned it in a system that reserved no special status for humanity, no place above nature. Some of the practices present in this issue assist in

What we usually call reality, aware of its multi-layered, veiled and even distorted condition, is a sort of pri for transfections. ${ }^{3}$ It is a network of heterogeneities, full of contact-experience forms, where we constitute one another. All actants change by combining and associating with other elements in a network of actants that increasingly evolves and mutates. An a priori, or the construct of it, has long since have always been inextricably entangled in associative networks that are increasingly complex, increasingly sympoietic or what we could call associative networks of sociotechnical collectives in Latour's terms. This topic is reflected in articles
such as "Disappearing bodies, disappearing objects: what such as "Disappearing bodies, disappearing objects: what
Years and Years can teach us about design" (Pandelakis 2021), which places the focus on producing large-scale scripts that go beyond the description of a specific use to recontextualise it in a network complex interactions. Some design practices involve a place from which it disappears. But can design practices help us abandon the
the problem of a conception of agency can be approached as imposed framework of representation? Or is its condition, an autonomous and monolithic subject. They think with the in terms of design, an unsolvable conflict?

material and open other possibilities to identify more hab-

itable policies and ontologies in the worlds in which we live

and cohabit. Some cases, such as "Mari Mutare" (Lorenzo

2021), serve as a positioned experimentation for a politic: of interdependence. These are practices that function as a
counterproposal and react to claims of immutability and
universality, sharing the concern about certain understandings of autonomy in terms of the exaltation of individuality.

TEMES DE DISSENY \#37
In fact, if Judith Butler's claim is taken into account, those other multi-agent relationships matter. The dible or silenced, among many others. But the visible and the invisible operate as a kind of complex framework where violence and power unfold. With regard to this issue, as the article "Co-designing Meaningful Dialogues on Sexual Harassment" notes (Benbrahim 2021), in order to reduce violence in terms of sexual harassment, we must - or we must at list try to - deconstruct the entire system that institutionalises invisible does not begin or end, it is always in the middle between things, inter-being(s), and operates as a kind of tency. Perhaps we could affirm that the invisible is per se, due to its condition, a conflict. Or maybe we could state that what is called in general terms visible-invisible occurs in a concatenation of conflictual layers, through the articulation of a cognitive, somatic and sensitive understanding, as it is approached in "The digital sublime: orientation strategies inous world" (López and Navarro 2021).

ible? What are the agencies for measuring and recording? Of course, in the contemporary context, the visible is no onger governed solely by the perceptual capacities of the as a specific agent. The multiplicity of technical and (centific artefacts developed over recent centuries has not implemented the vision spectrum of the human eye, but has come to glimpse where the eye cannot see as well as what the eye could not see. However, it should be noted The the masurement agent continues to be human. 作 semacy and instrumentalising ways of doing. Regarding

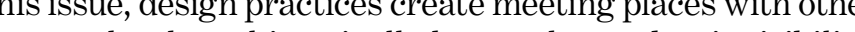
a that historically been relegated to invisibility, the subordinates that cease to be so when identifying place pexpression through the material. This non-anthropomorphic statement reveals representation as conflict yet again. As Hito Steyerl often points out, representation is a kind of is sappearance: the more something is represented, the more 3

Faced with this difficulty that affects, so to speak, the very preconditions of the actions of thought and design, the solu-

EDITORIAL it perpetuates the panoptic hegemony and perpetuates the erasure of the invisible, the made-invisible and the non-auand cultivates violence. In a similar way to the rhizome, the Therefore, a large part of these technocratic implementa- delegating technocratic solutions or even renouncing the of a given technical solution as they are understood as a very capacity for intervention. On the contrary, the solution comprehension of the relationships between different speeds f a more practical notion and implicit magnitudes in all contemporary socitechnica based on approaches such as, for example, the co-design to the scales of traditional physics, such as space and time: experiences analysed in the article "Between dissent and from the deep temporality of geological formation to the

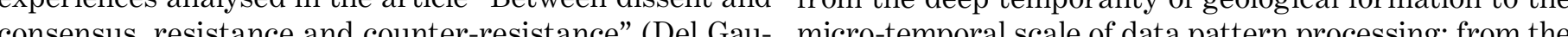
dio, Tanaka and Pastori 2021), or with a theoretical and space of particles, atoms and microbiological entities to the practical study of the context, strategies and tactics that construction of cumbersome infrastructures to transport operate or could operate there, which is what "Harnessing goods or colonise space. However, other types of scales can Conflict: Antagonism and Spatiotemporal Design Practices" also be imagined such as, for example, scales of affects or the proposes (Paez and Valtchanova 2021). This also includes construction of identity, understood as provisional condena reconfiguration of the theoretical field itself and the ca- sation points of instincts, social relations and technological pacity of new systemic thinking to profoundly affect the mediation. The whole body-mind can also be imagined - and perception of spaces for practical intervention, as seen in designed or counter-designed - as a point within a specthe article "A.I.R.: from radical individuality to connected trum of forces and speeds, which is what is analysed and subjectivity" (Cuesta, de la Torre and Arnaz 2021). In gener- dismantled in "It's Time to Relax: The critical importance of al, it does not involve demanding a life with less technology digital mental health products in the context of surveillance or with technology "on a human scale," but a different sort capitalism" (Partal and Smirnova 2021), a study of mental of technology with more shared agency between different health apps framed within the COVID-19 pandemic. actors on a planetary scale. It does not imply denouncing the manipulation carried out through the design of abusive interfaces, but ignoring, prohibiting and sabotaging any interface that is not understandable, auditable and configurable. Instead of fearing data and making a generic rejection of "surveillance capitalism" (Zuboff 2019), we are imagining systems in which our personal data is irrelevant, in which we are diluted instead of solidified, and thus the obsession with individualism is destroyed as a business model and transformation, performance, play and experimentation are fostered. It would also involve putting aside the naive notion of "defending nature" and understanding the planet of its relationships, for example, by updating issues such as sovereignty, artificiality or governance. Instead of demanding more insularity in the face of the threat of privatising global forces or technocratic governmental forces, it involves designing interconnection processes that defend collective rights on a global scale. Instead of fearing automatic processes and advocating a return to the "essential" of an "authentic" life or to the singularity of "unique" individuals, it means claiming another type of abstraction and design models that recognise forms of exploitation and material violence that have gone unnoticed until now.

This approach to invisible conflicts could be defined in terms of design on a planetary scale not because it responds to megalomaniacal projections of control, but because of the need for ideas, metaphors, procedures or the construction of scenarios that make it possible to imagine
the layers and irregular intermeshing of this growing tothe layers and irregular intermeshing of this growing to-
tality. Inevitably, this also means expanding the scope of tality. Inevitably, this also means expanding the scope of
design research, not to give even more weight to design as a decisive discipline but, on the contrary, to support alliances between knowledge and sensibilities and reduce the leading role of industrial production in favour of other types
of production, technology, rhythms, values and ways of life of production, technology, rhythms, values and ways of life that are also strategic. The practices and research that we associate with the notion of design on a planetary scale are, of
course, an expression of a sensitivity to issues of scale, but understood in a different way from the simple "scalability"

B. BRUSADIN / L. BENÍTEZ A subtly unpredictable aspect of projects that

address the planetary scale is the inevitability of reconguring the existing, rather than managing it, leading to n investigation of seemingly bizarre, paradoxical or even Deal" (Castillo-Vinuesa and Gankevich 2021). In this sense, magination on a planetary scale goes a step beyond what as been defined as speculative design over the last decade.

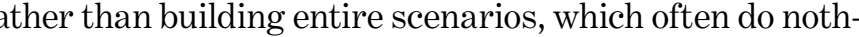
whe refect the inevitable frustration of trying to predict is too complex to predict, it involves inventing tentative serve our processes as if they were alien and unnatural.

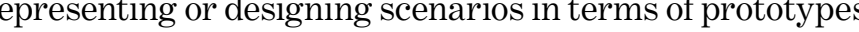
devices, infrastructures, situations or even institutions peans puting existing data and power structures under The effects can be counterintuitive or puzzling as co celerated intersection between layers of an interconnected planet and being able to take action within it.

Bani

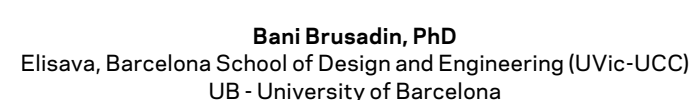

Bani Brusadin is a curator, educator and researcher. His research focuses on the creative
practices that intersect contemporary art, critical technologies, user cultures and the

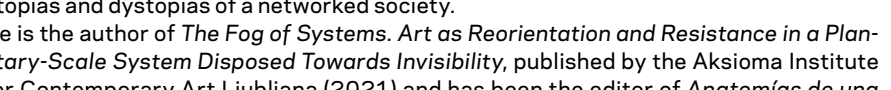

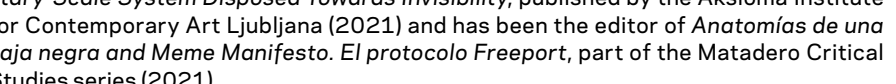

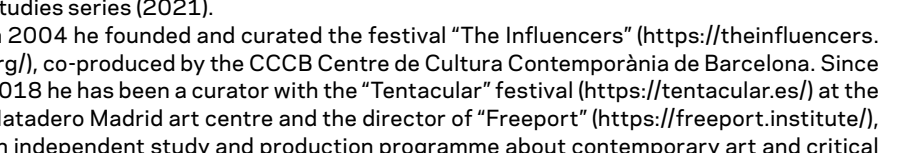

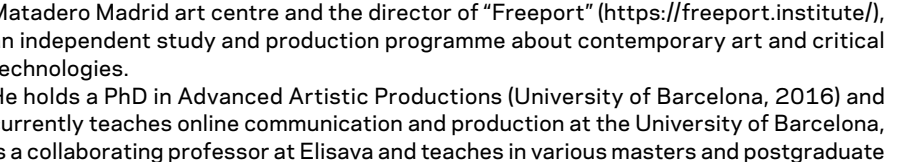
is a collaborating professor at Elisava and teaches in various masters and postgrad
programmes, including Digital Art Curatorship at ESDI and Data \& Design at Elisava. 


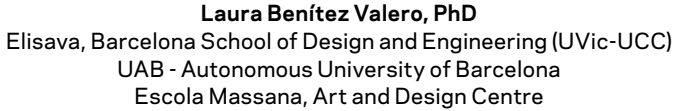

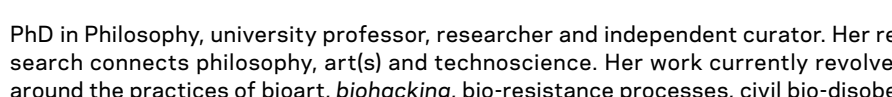
around the practices of bioart,
dience and non-human agents.

She is Head of Theory and Professoro of Critical and Cultural Studies in Arts and Design
Sscola Massanal. Professor of Technology and Design at Elisava and a Collaboratitg (Escola Massana), Professor of Technology and Design at Elisava and a Collaborating
Professor with the Master in Philiosophy for Contemporary Challenges, the Robotic She has been a guestresearcher with the Ars Electronica Centre and the MACBA Studies

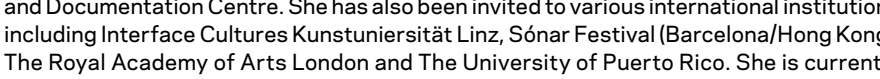

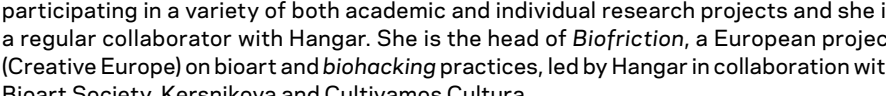

ENDNOTES

See, for example, "Internet Heal
other Mozilla Foundation reports.

Report 2020" (Alotta and Larsen 2021) and the

orts.

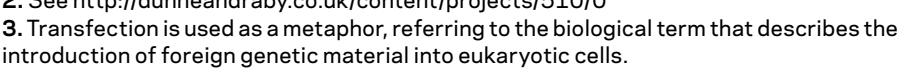

REFERENCES

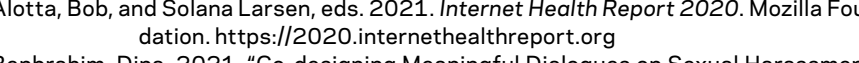

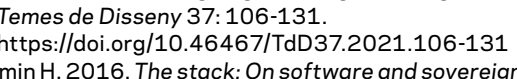

The MIT Press.

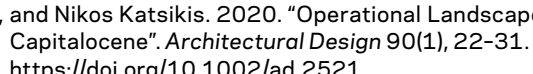

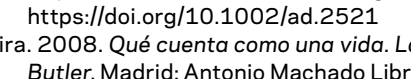

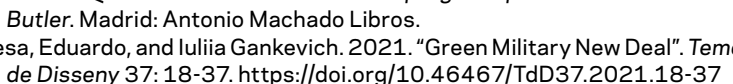

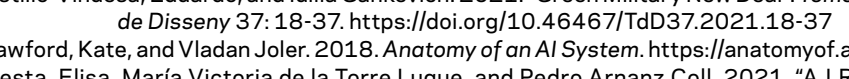

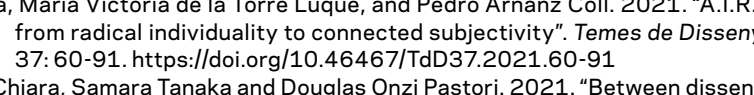

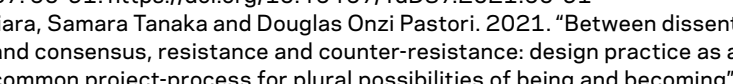
Tommon project-process for plural possibilities
Temes de Dissen $37: 156-181$.
https://doi.org/10.46467//TdD37.2021.156-181

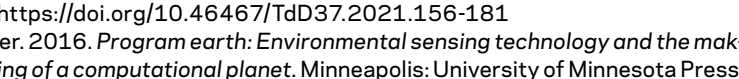

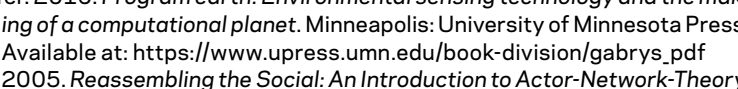

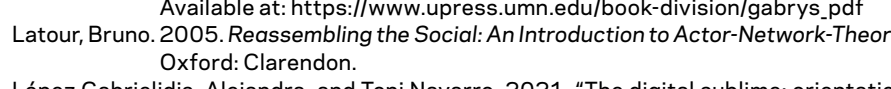

López Gabrielidisis, Alejandra, and Toni Navarro. 2021. "The digital sublime: orientation
strategies for a vertiginous world". Temes de Disseny 37: 226-243.

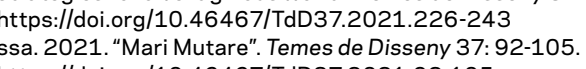

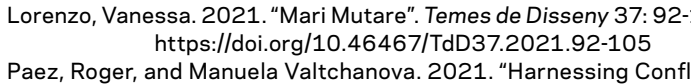

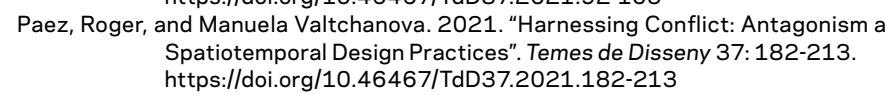

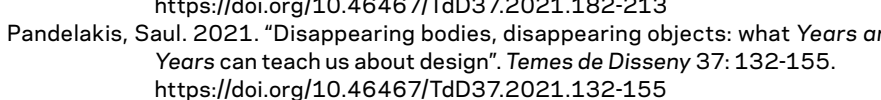

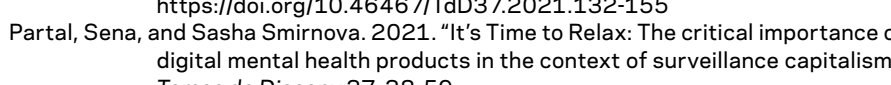
igital mental health products in the context of
Temes de Dissen $37: 38-59$.
Tttps://doi.org/10.46467/TdD37.2021.38-59

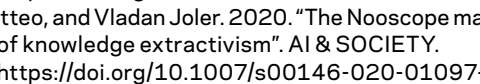

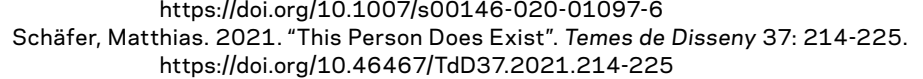

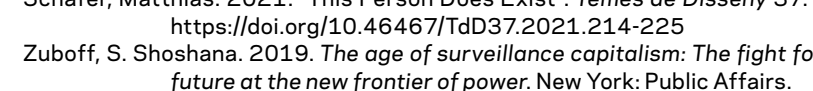

TEMES DE DISSENY \#37

L'experiència del planeta interconnectat és l'experiència d'una totalitat artificiali isovint sinistra. És el resultat de la la interseccióo acceleradad de diferents invisibles, que al seu torn competeixen entre si segons les loggiques irregulars d'interessos rivals, dissenys incompatibles, hảbits i irtmes humans, formes
de resistencia, errors, efectes collataterals i accidents. Per exemple, les jurisdiccions i les fronteres estect-laterals i accidents. Per exemple, les jurisdiccions i les fronteres estatals modernes estan sent traspassades per
paquets de dades i el seu processsament, que provooquen conflictes teòrics $i$ pràctics per la redefinició de la idea mateixa de sobirania, del seu subjecte del seu objecte (Bratton 2016). L'esfera personal es dilueix en plataformes dissenyades per a l'expressió de bilions d'usuaris amb diferents graus de
llibertat, però la seva autonomia colllapsa i s'esvaeix en infraestructures a una escala literalment inimaginable, rígidament propietàries però̀ ingovernables, automatitzades i alhora entròpiques, amb masses volàtils
d'usuaris i al mateix temps sostingudes per oligopolis globals, inversions estratègiques i itensions geopolítiques.'. Fins el regne del que és biològic del que és geològic són travessats per processos de quantificació, mode-
Itzacióo explotació ireformació que els transformen en camps de batalla entre e'extracció de recursos per a les activitats humanes i la producció de jectivitats expandides, o finsi tot de contrapoder (Gabrys 2016).
Que aixo formi part a ple dret de làmbit del disseny és evident si observem, per exemple, el funcionament d'un objecte tan ordinari avu dia com un centre de distribució d'Amazon. Els productes no hi esta característiques físiques apreciables a simple vista. Al contrari, en aques
colition magatzem entren en joc altres llocs $i$ altres cossos: els comportaments de consum dels usuaris a la plataforma, registrats $i$ analitzats pràcticament en
temps real; els patrons de funcionament de tots els actors en les cadenes distribució i logistica; i fins i tot, de manera indirecta, un model de negoci distribuciói ilogistica; i fins i tot, de maneral Amazon, com a productor, és capactac d'accedir a linstant a nínxols de mercat rendibles tan aviat com es detectin oportunitats de
beneficis (gràcies al rastreig immediat d'êxits o fracassos de venedors beneficis (gràcies al rastreig immediat d'èxits o fracassos de venedors
independents a la plataforma). Per descomptat, l'organització de l'espai independents a la plataforma). Per descomptat, 'organització de l'spai del sistema de gestió, que els guia a través d'un “paisatge operatiu” (Brenner i Katsikis 2020) construit pel i per al processament de dades en temps real. Utilitzen terminals i ells mateixos són terminals. L’objectiu d'aquesta
arquitectura - dissenyada, almenys en part, perquè s'adapti dinàmicament arquitectura -dissenyada, almenys en part, perquè s'adapti dinàmicament de manera dràstica la intel.ligència dels treballadors, el seu poder de decisio de manera drästica la intelel.ligència dels treballadors, el seu poder de decisis i se'n sap més aviat poc perquè són espais en efecte impenetrables en què
les visites guiades responen com a molt a finalitats de promoció. Són espais

EDITORIAL
12 comprensibles gràcies a operacions indirectes, com, per exemple, els relat
orals dels mateixos treballadors autoorganizats o l'enginyeria invers realitzada a partir de l'estudi de les patents registrades per Amazon o les seves empreses subsidiaries. El que ens interessa urbà mentre centenars de repartidors - generalment treballadors autònoms o falsos autònoms- recorren la ciutat seguint patrons impredictibles
pels planificadors urbans? Com afecta la vida urbana el fet que un nombre pels planificadors urbans? Com afecta la vida urbana el fet que un nombre
creixent d'actors prenguin decisions molt concretes motivades per les seves interaccions ambi ien plataformes privades (i dissenyades per permetre novalor i acumulació de capital estan sent sotmeses a un moviment tectònic
profund en el moment en què una activitat com pot ser conduir un vehicle, profund en el moment en què una activitat com pot ser conduir un vehicle,
que en general es considera treball no qualificat i sense valor afegit, ara

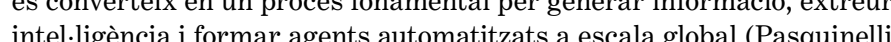
nerades mecànicament i agregades i no tant a escala individual, subjectiva
o particular- qüestionen procediments socials desenvolupats al llarg de dècades o segles, com, per exemple, la plas

la matexa noció de jurisdicció poritica?
En un món en què sabrecarrega d'estímuls cognitius en realitat empeny latenció de les persones cap a una plètora de detalls i reaccion a curt termini, les proporcions colossals dels procediments de quantificació i predicció es mouen realment cap al costat oposat de labstracció.
Els responsables d'aquesta deriva capa a labstracció són els processos de
producció i emmagatzematge de dades a escala massiva, però també el seu processament dinàmic i iescalable que genera patrons interpretatius
invisibles per als “usuaris" (exciutadans), sense comptar altres processos encara més incomprensibles - a vegades desordenats i fins i tot conflic-
tius- de calibratge, sincronització, traducció entre sistemes i protocols. tius- de calibratge, sincronitzacío, traducció entre sistemes i protocols
Tot això ens obliga a redefinir qüestions bàsiques de manera radicalment nova: com es pren una decisió? Com es defineix una estratègia - otàctica-
d'acció (individual i collectiva)? Com es replanteja la relació entre mon subjectius i planeta material? Què significa democràcia? I, de fet, com es
manifesta el conflicte? Com pot la recerca en disseny oferir instruments manifesta el conflicte? Com pot la recerca en disseny oferir instrument
per observar una era inestable de problemes sovint invisibles i a escal per observar una era inestable de problemes sovint invisibles i a escala
planetària? Com sorgeixen formes de resistència o de contradisseny si el
conflicte és a penes visible? 2

En làmbit del disseny, una multiplicitat de pràctiques no sols han abordat conflictes invisibles, sinó que han utilitzat la potència disruptiva del joc per generar disparadors crítics, que problematitzen les tàctiques d'esborrament
i invisisibilització, tant de conflictes com d'alteritats no humanes. Alguns dels referents fonamentals, en aquest cas des del disseny especulatiu, són
Dunne i Raby, que subverteixen la mediació tecnologica solucionista amb projectes com ara Foragers's i l'apliquen, irònicament, a l'agent responsable
de la desfeta ecològica: l'agent humà. Un agent concret, en el marc d' de la desfeta ecològica: l'agent humà. Un agent concret, en el marc d'un
sistema de producció i extracció concret, és a dir, capitalista. En aquest sistema de producció i extraccí́ concret, és a dir, capitalista. En aquest
número, la proposta operativa i experimental de "This Person Does Exist" numero, la proposta operativa experimental de
(Schäfer 2021) va precisament en aquesta direcció en desconstruir els (Schafer 2021 ) va precisament en aquestá direcció en desconstruir el
processos extractius implícits en la creació dels datasets necessaris per l'entrenament de sistemes de reconeixement facial.

El disseny contemporani ha contribuit a articular altres manere
de ser i estar amb la resta d'agents no humans i també a repensar, des de la materctialia del dissseny com a projecte-procoés comú per a les possibibilitats plurals de ser i arribar a ser. També es conformen pràctiques i projectes
que ajuden a comprendre conflictes relativament invisibles presents, per exemple, en la teoria contemporània. Cada vegada resulta més complex
continuar sostenint estructures dualistes com ara l'humà i el no humà, continuar sostenint estructures dualistes com ara l'humà i el no humà
l'artificial i i el natural o lorgànic i l'inorgànic. Sens dubte, fa temps que lartificial i el natural o l'orgànic i i inorgannic. Sens dubte, fa temps que
pensadors com Bruno Latour (2005) sostenen que això que anomenem món està tan fabricat que les einesi les tecnologies hi són tan omnipresents que no podem separar de manera significativa l'humà del no humà. Ara bé, cal

B. BRUSADIN / L. BENÍTEZ Per exemple, en quina mesura la proliferació de les compres en línia
està transformant de manera indirecta el ritme i la distribució del trànsit més el rol d'usuari)? Les mateixes nocions de treball, mitjans de producció, Joler 2020). De quina manera les dades -com a dades involuntàries, geo particular-qüestionen procediments socials desenvolupats al llarg de de ser i estar amb la resta d'agents no humansi t també a repensar, des de

questa igualtat en la diferenencia la que repta els agents humans a comprotectes en uns altres coestars, en altres relacions en què les escales, els localitzava i la recoll-locava en un sistema que no reservavav cap estatus dectiques presents en aquest número ajuden a aquesta relocalització des dexperimentació amb el que és matèric.

hixó que solem anomenar realitat, conscients de la seva condicic feccions ${ }^{3}$. Una xarra a d'heterogeneneitats, plenes de formes de contacte-expericia, on ens constitüm els uns als altres. Tots els actants es modifiquen en

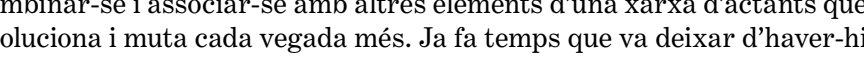
na priori o el constructe d'aquest. El Eque anomenem agents humansi i ino ada vegada més complexes, cada vegada més simpoiètiques, o el que en ermes de Latour podriem dir xarres associatives de coll.lectius socioteccrics.
questa quéstió queda reflectida en propostes com "Objectes i cossos que desapareixen: què ens pot ensennyar Years and Years sobre el disseny"
(Pandelakis 2021), en la qual s'aposta per produir guions a gran escala que vagin més enllà de la descripció d'un ús determinat per recontextualitzar-lo

Algunes pràctiques de disseny suposen un lloc des del qual abordar onoliticic. Pensen des del material i iobren altres possibilitats per atortiom 1 Jítiquesi iontogies més habitables en els mons que vivim que cortibuitem alguns casos, com a "Mari mutare" (Lorenzo 2021), serveixen com a xperimentació situada per a una política de la interdependència; pràcti'immutabilitat $i$ universalitat $i$ comparteixen la preocupació entorn de "El perill més grans per a mi és el perill del subjectece autónomi i monolítíc que intenta establir límits $i$ impermeabilitats absolutes, perquè aquest scial i la se que es nega a reconè em sembla que sobre aquesta mena de a no pot construir-se cap ètica o politica sòlites" (Jutler citada

De fet, si es té en compte l'afirmació de Judith Butler, aquestes al ja no pot ser únicament el centre de tota acció, ja que aquest perpetua Thegemonia panòptica, perpetua els esborraments dels no visibles, dels hvisibilitzats i dels no audibles o silenciats, entre molts altres. Però el visible potència s'esdevenen. A aquest propossit, tal com apunta larticle "Codisseny é diàlegs constructius sobre l'assetjament sexual" (Benbrahim 2021), si volem reduir la violència que comporta l'assetjament sexual, hem de desconstruir tot el sistema que institucionalitzai fomenta la violència, o, si més invisible no com una especicie de latência. Potser podríem afirmar que l'invisisible és per $e$ e per condicí, un conflicte. $O$ que, el que en termes generals anomenem visible-invisible es dona en una concatenació de capes conflictuals, per mitjà l'articulació d'un enteniment cognitiu, somàtic i sensible, com śaborda
"Sublim digital: estratègies d'orientació per a un món vertiginós" (López aro 2021).

Quins paràmetres operen en els règims del visible? Quines són les
cies de mesura i reg

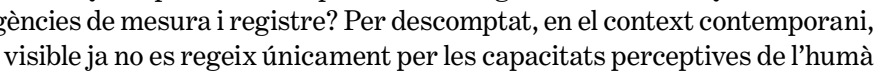
qualitat d'agent específic. La multiplicitat d'artefactes tecnocientífics a visió de 'lull humà, sinó que han arribat a albirar allà on l'ull no hi veu, però també el que 'ull no podia veure. Així i tot, cal remarcar que l'agent de
esura principal continua sent l'humài que, per tant, gran part d'aquestes mplementacions tecnocràtiques continuen operant des d'una noció d'excep-
cionalitat, supremacia i maneres de fer instrumentalitzadores. Respecte aquesta qüestió, les pràctiques de disseny generen llocs de trobada amb subalternes que deixen de ser-ho en articular llocs d'enunciació des del material. Una enunciació no antropomòrfica que, una vegada més, posa de 

ment estranyss, paradoxals o fins i tot controvertits, coma a "Green Military
New Deal" (Castillo-Vinuesa i Gankevich 2021). En aquest sentit, la imaDavant d'aquesta dificultat que afecta, per dir-ho així, les precondicions ginacía a escala planetària va un pas més enllà del que en lúltima dècada mateixes de l'acció de pensament $i$ disseny, la solució no consisteix a uti- s'ha anat definint com a disseny especulatiu. En lloc de construir escenaris renunciar a la capacitat mateixa d'intervenció. Al contrari, la solució és a l'hora d'intentar predir el que és massa complex per poder predir-se, es dotar-nos de punts de suport per a una nova configuració, adequada als tracta d'inventar mètodes temptatius per produir un efecte d'uncanny valley temps, de la noció pràctica d'agency quant a agència, eficàcia i capacitat invertit i observar els nostres processos com si fossin aliens i innaturals. d'accí,i i fer-ho amb plantejaments pràctics, com, per exemple, les experi- Representar-los o dissenyar-los quant a prototips de dispositius, infraes-
ències de codisseny analitzades a "Entre dissens i consens, resistèn

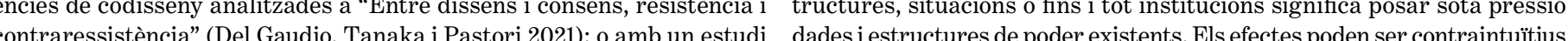
teoricopràctic del context $i$ de les estratègies $i$ tàctiques que hi operen o o desconcertants perquè uns fragments recognoscibles es recombinen en podrien operar, que és el que proposa “Approfitar el conflicte: antagonisme acoblaments improbables. Aquest tipus de procediment resulta clau per
i pràctiques de dissenyy espaciotemporals" (Paezi Valtchanova 2021). Fins capturar la intersecció accelerada entre capes d'un planeta interconnectat i tot amb una reconfiguració del mateix camp teòric ila capacitat d'un nou
pensament sistèmic d'afectar profundament la percepció dels espais d'in(Cuectivitat connectada" (Cuesta, de la Torre i Arnaz 2021). En general, no es tracta de reclamar una vida amb menys tecnologia 0 amb tecnologia "a escala humana", sinó una tecnologia diferent i amb més agència compartida
entre diferents actors a escala planetària. No es tracta de denunciar la d'ignorar, prohibiri i sabotejar tota interfície que no sigui comprensible, auditable i configurable. En lloc de la por de les dades i del rebuig genèric al "capitalisme de vigilància" "Zuboff 2019), imaginar sistemes en els qual
les nostres dades personals siguin irrellevants, en els quals els nostres jo es dilueixin en lloc de solidificar-se iaixíes destrueixi lindividualisme obsess com a model de negocici ies potenciil la transformació, la performance, e joc
l'experimentació. Es tractaria també de deixar de banda la noció ingènua de "defensa de la naturalesa" i comprendre el planeta com un conjunt de
naturocultures que requereix un redisseny radical de les seves relacions, naturocultures que requereix un redisseny radical de les seves relacions,
per exemple, en actualitzar questions com ara la sobirania, l'artificialitit per exemple, en actualitzar qüestions com ara la sobirania, l'artificicialitat
o la governança. En lloc de recclamar més insularititat davant lamenenaça de forces globals privatitzadores o forces governamentals tecnocraatiques, dissenyar processos d'interconnexió que defensin drets col-lectius a escala
global. En lloc de la por dels processos automàtics i d'advocar per un retorn a l'"essencial" "'una vida “autèntica" o a la singularitat d'individus " "ńnics",
reivindicar un altre tipus d'abstracció i dissenyar models que reconeguin
formes d'explotaciói i violència material fins ara desapercebudes. Es podria definir aquesta appoximació als conflictes invisibles en
termes de disseny a escala planetària, no perquè respongui a projeccions megalòmanes de control, sinó per la necessitat d'idees, metàfores, procediments o construcció d'escenaris que permetin imaginar les capes $i$ les soldadures irregulars d aquesta creixent totalitat. Inevitablement, això
també significa ampliar rabast de la recerca en disseny, no tant per donar
encerara més pes al disseny com a disciplina resolutiva, sinó al contrari. encara més pes al disseny com a disceplinan resolutiviva, sińó al contrar per fomentar aliances entre sabersi sensibilitats i reduir el protagonist
de la producció industrial a favor d'altres tipus - també estratègicsproductiques i la recerca que associem a la noció de disseny a e scala plane-
tària, per descomptat, són expressió d'una sensibilitat per les quiestion d'escala, però enteses d'una manera diferent de la simple "sccalabilitat"
d'unal determinada solucí tècnica, perquè s'entenen com una compren d'una determinada solució tècnica, perquè s'entenen com una comprensió
de les relacions entre diferents velocitats i magnituds implíities en tots els de les relacions entre diferents velocitats i magnituds implicites en tots els
processos sociotècnics contemporanis (Crawford i Joler 2018). Ens referim, temporalitat profunda de la formació geològica fins a l'escala miccost poral del processament de patrons de dades; des de lespai de partícules,

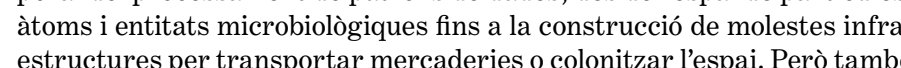
estructures per transportar mercaderies o colonitzar l'espai. Però també
es poden imaginar altres tipus d'escala, com, per exemple, la dels afectes es poden imaginar altres tipus d'escala, com, per exemple, la dels afectes
o de la construcció de la identitat, entesos com a punts de condensació
provisional d'instints, relacions socials i mediació tecnològica. El conjunt provisional d'instints, relacions socials imediació tecnologica. El conjunt
cos-ment també es pot imaginar -i dissenyar, o contradissenyar- com
un punt dins d'un espectre de forces i velocitats, que és el que s'analitza i

\section{Bani Brusadin, PhD
Elisava, Facultat de Dissenyi Enginineria de Barcelona (UVic-UCC)}

Bani Brusadinés comissari, docenti investigador. El seu àmbit de recerca són les pràcti-

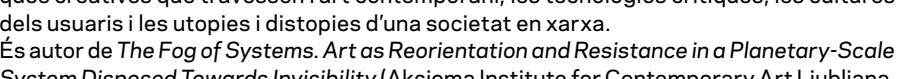
2021) i editor d'Anatomias de una coja negra i Meteme Manifesto. El protocolo Freeport

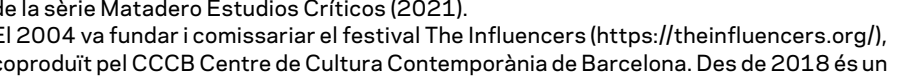
dels comissaris del Festival Tentacular (https://tentacular.es//) del centre dart Mataden

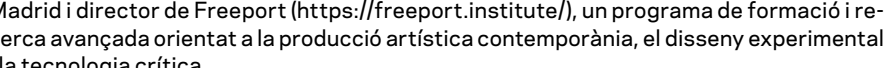
litecnologia criticica.
Es doctor en Produccions Artístiques Avancades (Universitat de Barcelona, 2016) iac-

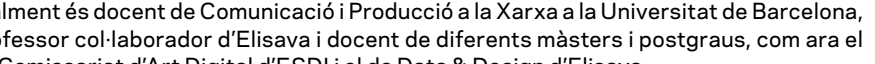

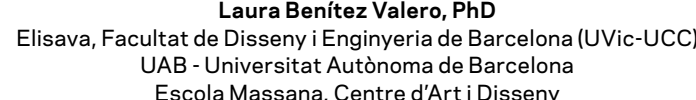

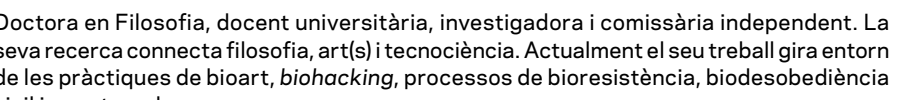
civili iagents no humans.
Es cap 'daraea de eeriai i rofessora d'Estudis Critics i Culturals al grau d'Arts i Disseny

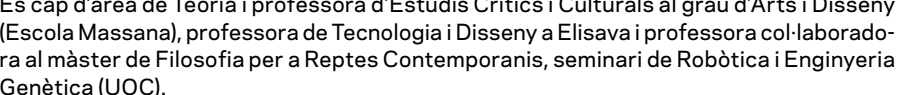
Ha estat investigadora convidada al Centre Ars Electronica ial Centre d'Estudisi D Docu-
mentaciód del MACBA. També ha estat convidada a diferents institucions internacionals

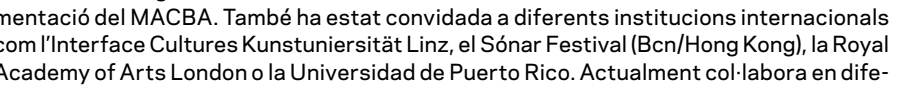

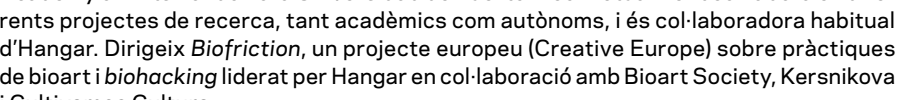
NOTES FINALS

1. Vegeu per exemple "Internet Health Report 2020" (Alotta i Larsen 2021) iels altres

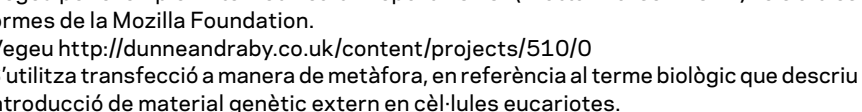
EDITORIAL mente a la e evolución de diferentes fact parte, para que salar la productividad al minimizar de manera drástica la inteligencia de los trabajadores, su poder Amazon, de los que se sabe más bien poco al ser espacios en efecto impepromoción. Son espacios comprensibles gracias a operaciones indirectas,
como, por ejemplo, los relatos orales de los propios trabajadores autoorga-
nizados o la ingeniería inversa realizada a partir del estudio de las patentes nizados o la ingeniería inversa realizada a partir del estudio de las patentes
registradas por Amazon o sus empresas subsidiarias. Lo que nos interesa ( está transformando de forma indirecta el ritmo y la distribución del tráfico urbano mientras cientos de repartidores - generalmente trabajadores
autónomos o falsos autónomos - recorren la ciudad siguiendo patrones impredecibles por los planificadores urbanos? ${ }^{2}$ Cono bana el hecho de que un numero creciente de actores tomen decisiones mas
concretas motivadas por sus interacciones con y en plataformas privadas (y
diseñadas diseñadas para no permitir otro rol que el de usuario)? Las mismas nociones
de trabajo, medios de producción, valor y acumulación de capital están siendo sometidas a un movimiento tectónico profundo en el momento en que una actividad como conducir un vehículo, que en general se considera
trabajo no cualificado y sin valor añadido, ahora se convierte en un proceso fundamental para generar información, extraer inteligencia y formar a
agentes automatizados a escala global (Pasquinelli y Joler 2020). iDe qué
manera los datos - no tanto a escala individual, subjetiva o particular, sino en cuanto a datos involuntarios, generados mecánicamente y agregadossiglos, como, por ejemplo, la planificicación urbana, el sindicalismo o la propia noción de jurisdicción politica?

En un mundo en el que la sobrecarga de estímulos cognitivos en realidad empuja la atención de las personas hacia una plétora de detalles y subjetividades expandidas, o incluso de contrapoder (Gabrys 2016). los procesos de producción y almacenamiento de datos a escala masiva,
La experiencia del planeta interconectado es la experiencia de una totalidad de diferentes ámbitos, cada uno con su topología articulada en subsistemas
y capas invisibles, que a su vez compiten entre sí según las lógicas irregula y capas invisibles, que a su vez compiten entre sí según las lógicas irregula-
res de intereses rivales, diseños incompatibles, hábitos y ritmos humanos, formas de resistencia, errores, efectos colaterales y accidentes. Por ejemplo, por paquetes de datos y su procesamiento, que provocan conflictos teóricos y de su objeto (Bratton 2016). La esfera personal se diluye en plataformas
diseñadas para la expresión de billones de usuarios con diferentes grados diseñadas para la expresión de billones de usuarios con differentes grados a una escalal literalmente inimaginable, rígidamente propietarias pero
ingobernables, automatizadas y a la vez entrópicas, con masas volátiles de usuarios y al mismo tiempo sostenidas por oligopolios globales, inversiones
estratégicas y tensiones geopoliticas.' Hasta el reino de lo biológicic y de lo
geológico son atravesados por procesos de cuantificacion, modelización geológico son atravesados por procesos de cuantificación, modelización,
explotación y reformación que los transforman en campos de batalla entre la extracción de recursos para las actividades humanas y la producción de Que esto forme parte a pleno derecho del ámbito del diseño es
evidente si observamos, por ejemplo, el funcionamiento de un objeto tan evidente si observamos, por ejemplo, el funcionamiento de un objeto tan
ordinario hoy en día como un centro de distribución de Amazon. Allí los productos no están organizados según una taxonomía comercial o funcional, ni siquiera por características físicas apreciables a simple vistas Al
contrario, en ese almacén entran en juego otros lugares y otros cuerpos: lo comportamientos de consumo de los usuarios en la plataforma, registrado y analizados prácticamente en tiempo real; los patrones de funcionamiento
de todos los actores en las cadenas de distribución y logistica; e incluso, de forma indirecta, un modelo de negocio flexible en el que Amazon, como
productor, es capaz de acceder al instante a nichos de mercado rentables tan pronto como se detecten oportunidades de beneficios (gracias al ras-
treo inmediato de éxitos o fracasos de vendedores independientes en la plataforma). Por supuesto, la organización del espacio diseñado tiene en plataforma). Por supuesto, la organizacion del espacio diseñado tiene en
cuenta la eficiencia de los movimientos de los trabajadores, pero existe un deslizamiento importante: los trabajadores siguen las instrucciones del sistema de gestión, que los guía a través de un “paisaje operativo" (Brenner
y Katsikis 2020) construido por y para el procesamiento de datos en tiempo

B. BRUSADIN / L. BENÍTEZ

de cuantificación y predicción se mueven realmente hacia el lado opuesto
de la abstracción. Los responsables de esta deriva hacia la abstracción son pero también su procesamiento dinámico y escalable que genera patrones otros pros án más incomprensibs a veces desordes sin contar otros procesos aún más incomprensibles - a veces desordenados y hast
conflictivos- de calibración, sincronización, traducción entre sistemas y rotocolos. Todo esto nos obliga a redefinir cuestiones básicas de manera adicalmente nueva: ¿cómo se toma una decisión? ¿Cómo se define una strategia - otáctica- de acción (individual y colectiva)? ? íómo se replan-
ea la relación entre mundos subjetivos y planeta material? ¿Qué significa democracia? Y, de hecho, iccómo se manifiesta el conflicto? ¿Cómo puede la nvestigación en diseño ofrecer instrumentos para observar una era inesta-
le de problemas a menudo invisibles y a escala planetaria? ¿Cómo surgen rmas de resistencia o de contradiseño si el conflicto es apenas visible?

En el ámbito del diseño, una multiplicidad de prácticas no solo han abor-
dado conflictos invisisbles sino que han utilizado la potencia disruptiva del Jus disparadores críticos, que problematizus lastiva del de borrado e invisibilización, tanto de conflictos como de alteridades no humanas. Algunos de los referentes fundamentales, en este caso desde tecnológica solucionista con proyectos como Foragers' ${ }^{2}$ y la aplican, irónicamente, al agente responsable de la debacle ecologica: el agente humano. concreto, es decir, capitalista. En este numero, la propuesta operativa y
eperimental de "This Person Does Exist" (Schäfer 2021) va precisamente n esta dirección al deconstruir los procesos extractivos implíitos en la conocimiento facial.

er y estar con el restomo de agentes ha no humananos y también a repenssar, desde la materiarian resto de agentes no humanos y también a repensar, desde 
cuerpos que desaparecen: lo que Years and Years puede enseñarnos sobre operan o podrían operar, que es lo que propone "Aprovechar el conflicto:
el diseño" (Pandelakis 2021), en la que se apuesta por producir guiones a antagonismo y prácticas de diseño espaciotemporales" (Paez y Valtcha-

gran escala que vayan más allá de la descripción de un uso determinado nova 2021). Incluso con una reconfiguración del propio campo teórico y la

para recontextualizarlo en una red de interacciones complejas.
Algunas prácticas de diseño suponen un lugar desde el que abordar la percepción de los espacios de intervención práctica corofundamente

el problema de una concepción de agencia en calidad de sujeto autónomo "A.I.R.: de la individualidad radical a la subjetividad conectada" (Cuesta, y monolitico. Piensan desde el material y abren otras posibilidades para de la Torre y Arnaz 2021). En general, no se trata de reclamar una vida con articular políticas y ontologías más habitables en los mundos que vivimos, menos tecnología o con tecnología "a escala humana", sino una tecnología
que cohabitamos. En algunos casos, como en "Mari mutare" (Lorenzo distinta y con más agencia compartida entre diferentes actores a escala que cohabitamos. En algunos casos, como en "Mari mutare" (Lorenzo distinta y con más agencia compartida entre diferentes actores a escal dependencia. Prácticas a modo de contrapropuesta que reaccionan ante las vés del diseño de interfaces abusivas, sino de ignorar, prohibir $y$ saboter pretensiones de inmutabilidad y universalidad y comparten la preocupación toda interfaz que no sea comprensible, auditable y configurable. En lugar en torno a ciertas comprensiones de la autonomia en cuanto a la exaltación del miedo a los datos y del rechazo generico al "capitalismo de vigilancia" de la individualidad. "El mayor peligro para mí es el peligro del sujeto au- （Zuboff 2019), imaginar sistemas en los que nuestros datos personales sean

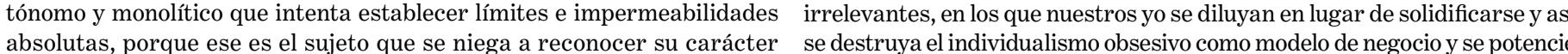
fundamentalmente social y su interdependencia. Y me parece que sobre la transformación, la performance, el juego y la experimentación. Se tratart este tipo de base no puede construirse ninguna ética o política sólidas" también de dejar de lado la noción ingenua de "defensa de la naturaleza" (Judith Butler citada en Burgos 2008, 410).
De hecho, si se toma en cuenta la afirmación de Judith Butler, esas
un redisendor radical de sus relaciones, por ejemplo, al actualizar cuestione De hecho, si se toma en cuenta la afirmación de Judith Butler, esas un rediseño radical de sus relaciones, por ejemplo, al actualizar cuestiones
otras relaciones entre agentes múltiples importan. El régimen de lo visual
como la soberanía, la artificialidad o la gobernanza. En lugar de reclamar otras relaciones entre agentes multiples importan. El régimen de lo visual como la soberaniá, la artificialidad ola gobernanza. En lugar de reclama
ya no puede ser únicamente el centro de toda acción, ya que este perpetúa más insularidad ante la amenaza de fuerzas globales privatizadoras o la hegemonía panóptica, perpetúa los borrados de los no visibles, de los fuerzas gubernamentales tecnocráticas, diseñar procesos de interconexión invisibilizados y de los no audibles o silenciados, entre muchos otros. Pero que defiendan derechos colectivos a escala global. En lugar del miedo a los lo visible y lo invisible operan como una suerte de entramado complejo, procesos automáticos y de abogar por una vuelta a lo "esencial" de una vida
donde violencia y potencia acontecen. A este respecto, tal como apunta "auténtica" o a la singularidad de individuos “únicos", reivindicar otro tipo

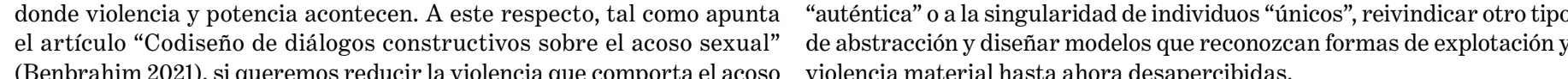
sexual, debemos deconstruir todo el sistema que institucionaliza y fomenta la violencia, o al menos debemos intentarlo. De un modo similar al rizoma, lo
invisible no empieza ni acaba, siempre está en el medio, entre las cosas, ininvisible no empieza ni acaba, siempre está en el medio, entre las cosas,
tersereses) yopera como una especie de latencia. Quizás pudiéramos afirm que lo invisible es per se, por condición, un conflicto. O que eso denominado
en términos generales visible-invisible se da en una concatenación de capas conflictuales, a través de la articulación de un entendimiento cognitivo, somatico y sensible, como se aborda en “Lo sublime digital: estra
orientación para un mundo en vértigo" (López y Navarro 2021).

y magnitudes implícitas en todos los procesos sociotécnicos contemporá-
neos (Crawford y Joler 2018). Nos referimos, pues, a las escalas de la fisica tradicional, como el espacio y el tiempo: desde la temporalidad profunda de la formación geoológica a la escala microtemporal del procesamiento
de patrones de datos; desde el espacio de partículas, átomos y entidades microbiológicas hasta la construcceión de engorrosasas infraestructuras para
transportar mercancías o colonizar el espacio. Pero también se pueden imaginar otros tipos de escala, como, por ejemplo, aquella de los afectos o de la construcción de la identidad, entendidos como puntos de condensación provisional de instintos, relaciones sociales y mediación tecnológica. conjunto cuerpo-mente también se puede imaginar -y diseñar, o contra-
diseñar- como un punto dentro de un espectro de fuerzas y velocidades, que es lo que se analiza y desmonta en "Es momento de relajarse: la gran importancia de los productos de salud mental en el contexto del capitalismo de vigilancia" (Partal y Smirnova 2021), un estudio

mental en el contexto de la pandemia de COVID-19. la escala planetaria es la inevitabilidad de reconfigurar lo existente, en lugar de gestionarlo, lo que da lugar a una investigación de ensamblajes aparentemente extraños, paradójicos o incluso controvertidos, como en
"Green Military New Deal" (Castillo-Vinuesa y Gankevich 2021). En este sentido, la imaginación a escala planetaria va un paso más allá de lo que de construir escenarios completos, que a menudo no hacen otra cosa que reflejar la frustración inevitable a la hora de intentar predecir lo que e demasiado complejo para poder predecirse, se trata de inventar método tentativos para producir un efecto de uncanny valley invertido y observar nuestros procesos como si fueran ajenos e innaturales. Representarlo
o diseñarlos en cuanto a prototipos de dispositivos, infraestructuras, situaciones o incluso instituciones significa poner bajo presión datos y es-
tructuras de poder existentes. Los efectos pueden ser contraintuitivos o desconcertantes porque unos fragmentos reconocibles se recombinan e ensamblajes improbables. Este tipo de procedimiento resulta clave para
capturar la intersección acelerada entre capas de un planetaintereonectado y poder intervenir en ell.

BIOGRAFAS

Bani Brusadin, PhD
Elisava, Facultad de Diseño Ingenieria de Barcelona (UVic-UCC)
UB - Universidad de Barcelona

Bani Brusadin es comisario, docente e investigador. Su ámbito de investigación son las
prácticas creativas que atraviesan el arte contem poráneo, las tecnologias criticas, las

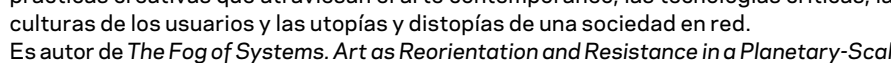

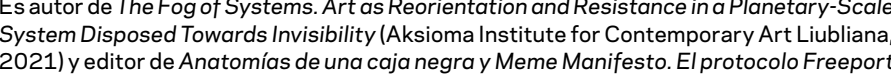

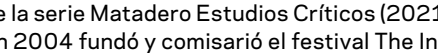

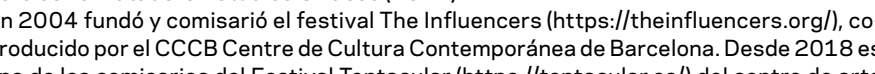
uno de los comisarios del Festival Tentacular (https://tentaculareses/) del centro de arte formación einvestigación avanzzada orientado a la producción artística contemporánea,
eld diseño experimental y la tecnologicia critica.
Es doctor en Producciones Artísticas Avanzadas (Universitat de Barcelona, 2016) y EEd doctor en Producciones Artisticas Avanzadas (Universitat de Barcelona, 2016) y
actualmente es docente de Comunicacion y Producción en la Universitat de Barcelona

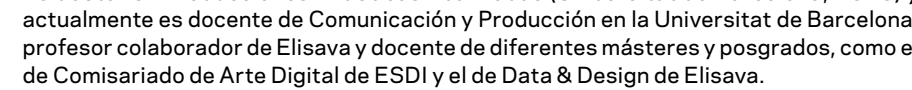

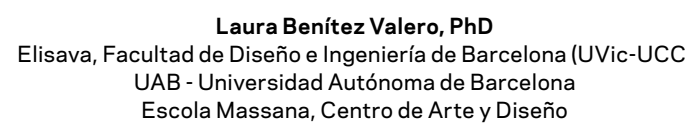

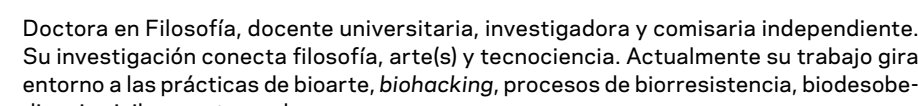

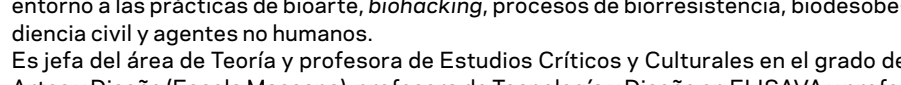

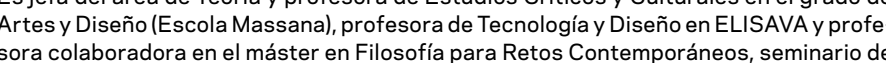

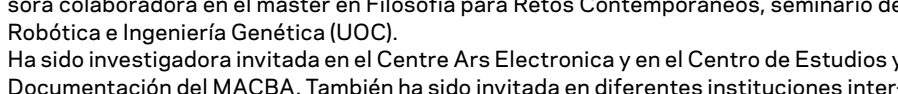

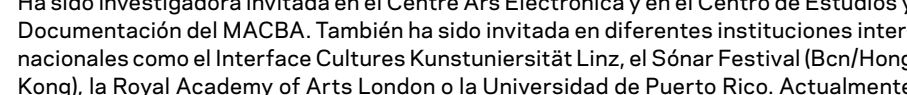

B. BRUSADIN / L. BENÍTEZ

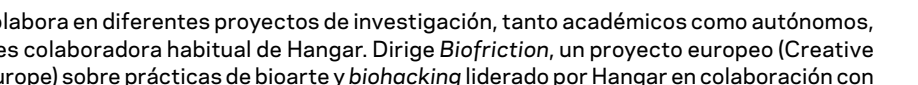

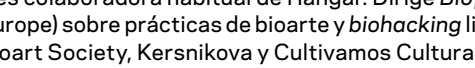

NOTAS FINALES

\section{Véanse por ejemplo "Internet Healt
informes de la Mozilla Foundation.
2.Véase}

th Report 2020" (Alotayl

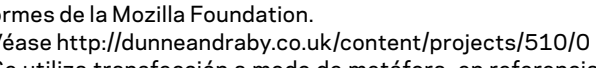

aby.co.uk/content/projects/510/0 REFERENCIAS

Ver listado completo de referencias en la página 12. 